\title{
Development of $x$-ray radiography for high energy density physics
}

Cite as: Phys. Plasmas 21, 102712 (2014); https://doi.org/10.1063/1.4900867

Submitted: 04 July 2014 . Accepted: 13 October 2014 . Published Online: 31 October 2014

A. Morace, L. Fedeli, D. Batani, S. Baton, F. N. Beg, S. Hulin, L. C. Jarrott, A. Margarit, M. Nakai, M. Nakatsutsumi, P. Nicolai, N. Piovella, M. S. Wei, X. Vaisseau, L. Volpe, and J. J. Santos

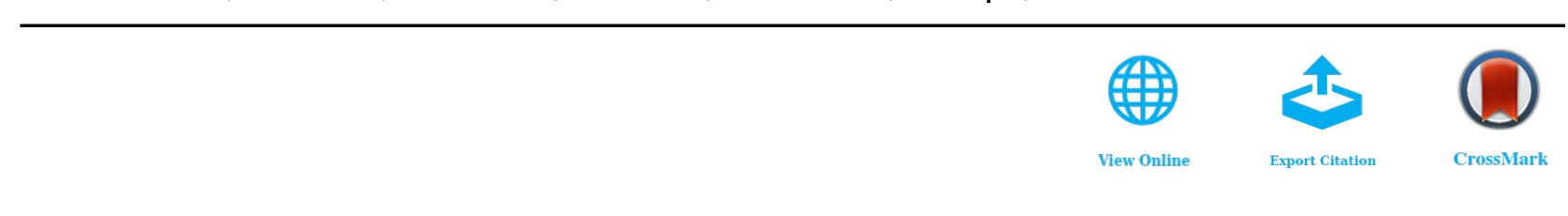

\section{ARTICLES YOU MAY BE INTERESTED IN}

X-ray source studies for radiography of dense matter

Physics of Plasmas 16, 033101 (2009); https://doi.org/10.1063/1.3076207

High-energy Ka radiography using high-intensity, short-pulse lasers

Physics of Plasmas 13, 056309 (2006); https://doi.org/10.1063/1.2178775

High-energy (>70 keV) x-ray conversion efficiency measurement on the ARC laser at the National Ignition Facility

Physics of Plasmas 24, 033112 (2017); https://doi.org/10.1063/1.4978493 


\title{
Development of x-ray radiography for high energy density physics
}

\author{
A. Morace,${ }^{1,2}$ L. Fedeli, ${ }^{1, a)}$ D. Batani, ${ }^{1}$ S. Baton, ${ }^{3}$ F. N. Beg, ${ }^{4}$ S. Hulin, ${ }^{1}$ L. C. Jarrott, ${ }^{4}$ \\ A. Margarit, ${ }^{1}$ M. Nakai, ${ }^{2}$ M. Nakatsutsumi, ${ }^{3}$ P. Nicolai, ${ }^{1}$ N. Piovella, ${ }^{5}$ M. S. Wei, ${ }^{6}$ \\ X. Vaisseau, ${ }^{1}$ L. Volpe, ${ }^{1}$ and J. J. Santos ${ }^{1}$ \\ ${ }^{1}$ University Bordeaux, CNRS, CEA, CELIA (Centre Lasers Intenses et Applications), UMR 5107, F-33405 \\ Talence, France \\ ${ }^{2}$ ILE, Osaka University, 2-6 Yamadaoka, Suita City, Osaka, Japan \\ ${ }^{3}$ LULI, UMR 7605 CNRS-CEA-Ecole Polytechnique, Palaiseau, France \\ ${ }^{4}$ Center for Energy Research, University of California, 9500 Gilman Drive, La Jolla, California 92093, USA \\ ${ }^{5}$ Dipartimento di Fisica, Università di Milano, Via Celoria 16, 20133 Milan, Italy \\ ${ }^{6}$ General Atomics, 3550 General Atomics Court, San Diego, California 92121, USA
}

(Received 4 July 2014; accepted 13 October 2014; published online 31 October 2014)

We describe an experiment performed at the LULI laser facility using an advanced radiographic technique that allowed obtaining 2D, spatially resolved images of a shocked buried-code-target. The technique is suitable for applications on Fast Ignition as well as Warm Dense Matter research. In our experiment, it allowed to show cone survival up to Mbar pressures and to measure the shock front velocity and the fluid velocity associated to the laser-generated shock. This allowed obtaining one point on the shock polar of porous carbon. (C) 2014 AIP Publishing LLC.

[http://dx.doi.org/10.1063/1.4900867]

\section{INTRODUCTION}

High Energy Density Physics (HED) is a very active research field, which developed in recent years. ${ }^{1}$ By definition, HED states correspond to an energy density $\geq 10^{11} \mathrm{~J} / \mathrm{m}^{3}$ or correspondingly pressures exceeding $1 \mathrm{MBar}$. The study of such physical systems is of great interest for many branches of Physics, including Astrophysics, Material Science, and Inertial Confinement Fusion (ICF) research. ${ }^{2-4}$ Densities and temperatures characteristic of HED states are typical of many astrophysical objects including the cores of giant planets, supernova explosions, and stellar astrophysics. Many striking phenomena appear in this regime, including interesting phase transitions with important consequences. For instance, in planetary physics, carbon subject to very high pressures in the mantle of giant planets can metallize, leading to high electric conductivity, which, via dynamo effect, could explain the origin of the large observed magnetic fields in planets like Uranus and Neptune. ${ }^{5}$

Nowadays, it is relatively easy to produce HED states by focusing high power lasers on matter, driving multimegabar shocks and being able to compress the matter (in implosions) up to almost 1000 times solid density. ${ }^{6}$ These extreme states of matter are also very important for the study of strongly coupled, partially or fully degenerate plasmas with applications to ICF and its alternative approaches such as Fast Ignition $(\mathrm{FI})^{7}$ or shock ignition. ${ }^{8}$

In FI, HED states are produced both as a consequence of the "normal" compression phase and as a result heating induced in matter by the propagation of an intense beam of fast (relativistic) electrons in the final "ignition" phase. One promising approach to FI is the use of the cone-in-shell target geometry. ${ }^{9}$ Indeed the cone keeps a clear access for the

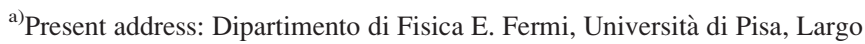
Bruno Pontecorvo 3, 56127 Pisa, Italy.
}

ultra-intense beam from the ablation plasma produced during implosion, which allows generating the fast electron beam very close to the compressed fuel core. Of course the cone material (usually gold and more recently diamond ${ }^{10}$ ) will undergo a transition to HED states when crossed by the fast electron beam. In turn, the electric conductivity and collisionality of the heated cone material will influence the propagation of fast electrons. ${ }^{11}$ An additional, still open issue is represented by cone survival during shell implosion, which may be compromised by the huge pressures, driving strong shocks and plasma jets through the cone walls and the cone tip. $^{12}$

Diagnosing these new HED physical regimes represents a very challenging task. In particular, it is important being able to diagnose the density and temperature profiles in rapidly transient phenomena such as shock propagation in matter, providing information on the target conditions at different times, allowing to study the dynamics of such complex physical phenomena.

In this work, we describe the optimization of $\mathrm{x}$-ray point projection radiography, a technique allowing to obtain 2Dspatially and time-resolved images with applications ranging from FI research to the determination of equations of state (EOS). In particular, such diagnostics can be of interest in Shock Ignition experiment where one of the most used diagnostics (VISAR ${ }^{13}$ ) is often "blinded" by the preheating caused by the laser pulse. ${ }^{14}$ Of course, in literature is possible to find several works related different methods and applications of $\mathrm{x}$-ray radiography techniques to HED plasmas. Early applications of $\mathrm{x}$-ray radiography, dating before the advent of Chirped Pulse Amplification (CPA) laser systems and still widely used now, make use of extended x-ray sources coupled to imaging optics and $\mathrm{x}$-ray streak cameras, providing temporally resolved $\mathrm{x}$-ray radiographies. ${ }^{15}$ Of course, the main limitation of this technique is the intrinsic 1dimensional spatial resolution recorded by streak cameras. 
The imaging system may be simple pin-hole cameras or more complex Kirkpatrick Baez microscopes or similar type of x-ray optics (see Ref. 16 for instance). A more recent technique employing long pulse laser systems and large foil backlighters is characterized by the implementation of multiple pin-hole array coupled with $\mathrm{x}$-ray framing camera. This technique allows obtaining $2 \mathrm{D}$ spatially and temporally resolved $\mathrm{x}$-ray radiographies, with temporal resolution $\geq 50$ ps. ${ }^{17}$ Although this is quite powerful, the use of pin-holes sensibly reduces the $\mathrm{x}$-ray flux available for radiography and requires therefore a large laser energy to create an intense backlighter, as well as a very accurate design of the x-ray ray shielding to reduce the background level in harsh x-ray and $\gamma$-ray environment. Radiographies have also been obtained by imaging the target with $\mathrm{x}$-ray optics such as a spherically bent Bragg crystals, allowing in this case also a precise selection of the $\mathrm{x}$-ray wavelength. ${ }^{18}$ The use of crystals for imaging can provide highly spatially resolved $(\sim 15-20 \mu \mathrm{m})$ monochromatic images, but, again, at the same time strongly reduces the $\mathrm{x}$-ray flux on the detector, due to the low reflectivity of Bragg crystals $\left(\sim 10^{-4}\right)$.

After the advent of CPA, short pulse ( $\sim$ ps) lasers have been used to generate X-rays short pulses, as used in our work, allow to drastically reduce the temporal resolution, since the $\mathrm{x}$-ray source duration is directly correlated to the laser pulse duration (or just a few ps longer due to the time needed by fast electrons to cool down in the foil). Dynamic evolution can be then obtained in multiple shots by varying the delay between the ps and the ns pulses.

Another powerful technique to diagnose HED plasmas is Compton point-projection radiography, which provides high resolution (close to the diameter of the backlighter wire) for very high-density plasmas. ${ }^{19}$ However, the backlighter x-ray energy range from 60 to $200 \mathrm{keV}$ requires very high plasma areal densities to be efficiently applied, densities obtainable at the moment only at very large facilities such as Omega laser at the Laboratory of Laser Energetics in Rochester and at the Lawrence Livermore National Ignition Facility.

In the present work, applications of x-ray point projection radiography technique, providing at the same time high spatial resolution to high x-ray flux are discussed. The work presented here was a preparatory stage of an experiment addressed to study fast electron generation and transport in shock driven buried cone targets. ${ }^{20}$ It was aimed at characterizing the propagation of the shock into a buried cone target, and at evaluating cone survival in presence of a strong shock. This kind of target represents a perfect sample for $\mathrm{x}$-ray radiography optimization, since many details, as well as the shock front itself, can be imaged allowing evaluating resolution, intensity and contrast of the radiographies.

As said before, we used buried cone targets in order to test the hydrodynamics and cone survival in FI. Of course, in a realistic FI scenario the conditions are quite different since the capsule is subject to implosion, resulting in high pressures on the cone wall and driving a plasma jet through the cone tip. In our case instead, a strong planar shock counterpropagates with respect to the cone axis. Despite the differences from a realistic FI scenario, it is still possible to evaluate the cone survival undergoing a strong shock and study the fast electron generation in modified laser-plasma interaction (LPI) conditions, in event of shock breakout, or the fast electron transport through a shocked material, depending on the delay between the ps and the ns pulses.

\section{EXPERIMENTAL SETUP}

The experiment was performed at LULI2000 laser facility of the Laboratoire pour l'Utilisation des Lasers Intenses (LULI). For the experiment, we have used two laser beams: a long pulse one and a short pulse one. The first one is able to deliver up to $1 \mathrm{~kJ}$ of laser energy in $5 \mathrm{~ns}$ at $1053 \mathrm{~nm}(1 \omega$ frequency), and the second one, thanks to the CPA technique, can be compressed to 1 ps pulse duration, $\sim 70 \mathrm{~J}$ pulse energy at $1 \omega$. In the present experiment, the ns and the compressed pulse were frequency doubled, delivering respectively $\sim 500 \mathrm{~J}$ in $5 \mathrm{~ns}$ square temporal profile on a $500 \mu \mathrm{m}$ spot size, optically smoothed by means of a random phase plate, and $\sim 15 \mathrm{~J}$ in 1 ps on a $10 \mu \mathrm{m}$ spot size.

The targets used during the experiment are relatively complex: they were constituted by copper-coated carbon buried cone in a multilayer target (see Fig. 1). The cone had a depth of $400 \mu \mathrm{m}$, a $17^{\circ}$ half angle aperture, and $50 \mu \mathrm{m}$ cone tip size and it was coated with $10 \mu \mathrm{m} \mathrm{Cu}$. The carbon layer, with density of $2 \mathrm{~g} / \mathrm{cm}^{3}$, extended $100 \mu \mathrm{m}$ beyond the cone tip, followed by $10 \mu \mathrm{m}$ silver (Ag) layer and finally by $6.5 \mu \mathrm{m} \mathrm{CH}$ plastic as ablator. The ns pulse was focused on the $\mathrm{CH}$ plastic ablator with an incidence of $22.5^{\circ}$ to drive a strong shock in the target material. General Atomics, San Diego, produced the targets.

Two main diagnostics have been implemented during the experiment. A streaked optical pyrometer (SOP), looking at the cone, was used to characterize the shock and in particular to determine the shock breakout time from the cone tip.

The second diagnostic was an instantaneous X-ray radiography. The ps laser beam was focused on a $10 \mu \mathrm{m} \mathrm{Cu}$ wire to perform $\mathrm{x}$-ray point projection radiography to characterize the shock propagation at different time delay (see Fig. 2) by varying the delay between the ns and the ps beams. The laser beam was tightly focused onto the wire to produce an intense $\mathrm{x}$-ray source at $8 \mathrm{keV}$ dominated by $\mathrm{K} \alpha$ emission produced by the interaction between $\mathrm{Cu}$ atoms and the fast electrons generated by the short-pulse high-intensity laser.

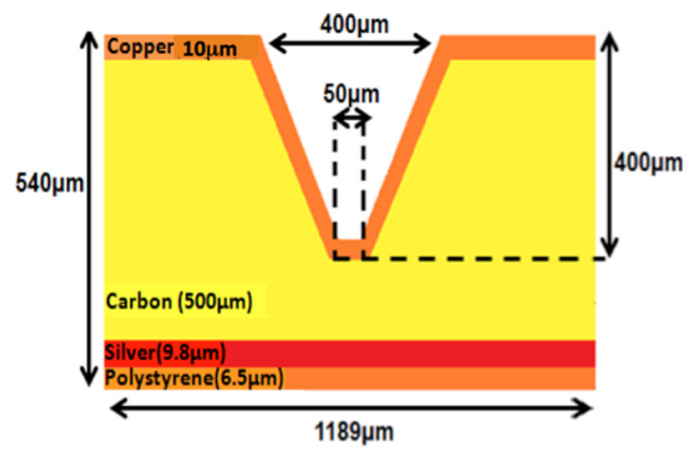

FIG. 1. Scheme of the target used in the experiment. The Cu layer thickness at cone tip was $23 \mu \mathrm{m}$. 


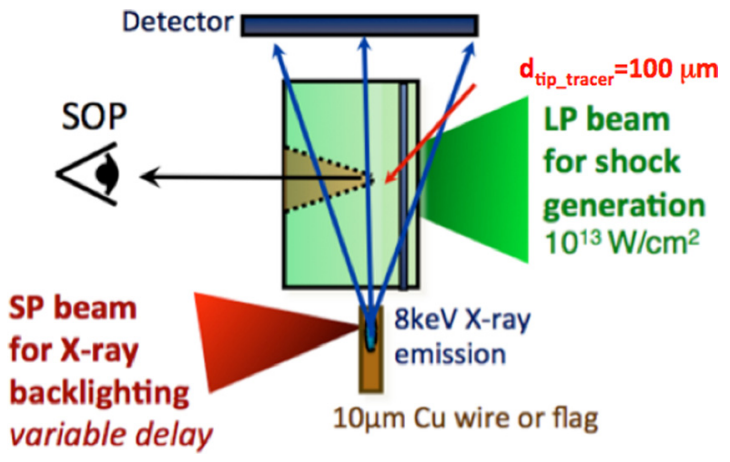

FIG. 2. Layout of the experimental setup (in reality the ns beam was incident at $22.5^{\circ}$ ).

\section{POINT PROJECTION X-RAY RADIOGRAPHY SETUP}

The $\mathrm{x}$-ray point projection radiography setup is represented in Fig. 2.

The $10 \mu \mathrm{m} \mathrm{Cu}$ wire was aligned normally to the cone axis at a distance of $5 \mathrm{~mm}$ correspondingly to the cone tip position. The $\mathrm{x}$-rays were collected by a Fujifilm BAS-MS imaging plate (IP) placed at $20 \mathrm{~cm}$ from the target providing a magnification of $\sim 40 \times$.

The $\mathrm{x}$-ray source was characterized by disposing $\mathrm{Al}$ foils with different thicknesses on the detector. The single $\mathrm{Al}$ layer thickness was $15.6 \mu \mathrm{m}$ and the stack thickness was sequentially increased from $2 \times$ to $10 \times$ as shown in Fig. 3 .

The Al stacks were aligned along the vertical direction, following the backlighter orientation. The experimental results (see Fig. 3 down) are perfectly interpolated assuming a spectrum given by the $\mathrm{Cu} \mathrm{K} \alpha$ emission line (i.e., photon energy of $8 \mathrm{keV}$ ) and a hard $\mathrm{x}$-ray contribution coming from
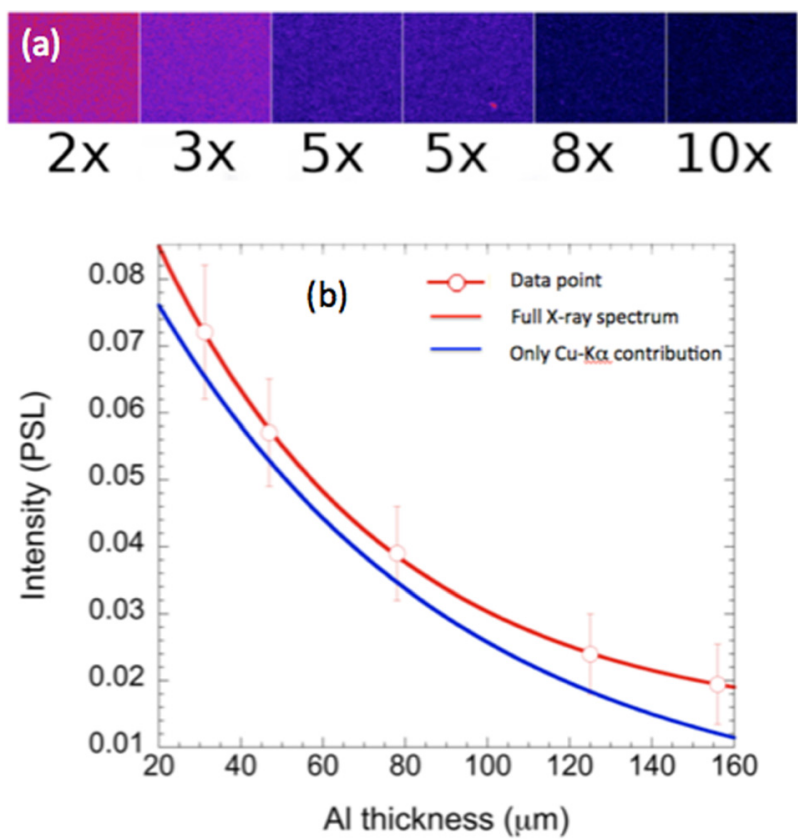

FIG. 3. (a) Al steps for $\mathrm{x}$-ray source calibration $(1 \times=15.6 \mu \mathrm{m})$; (b) Measured intensity on IP and fit (solid line) obtained using a spectrum composed by the $\mathrm{K} \alpha$ line plus a bremsstrahlung component (red line). Also shown the $\mathrm{K} \alpha$ contribution alone (blue line). In this case, the slope corresponds to the mass absorption coefficient using the absorption coefficient given by NIST for a pure $\mathrm{Cu}-\mathrm{K} \alpha$ emission at $8 \mathrm{keV}$.

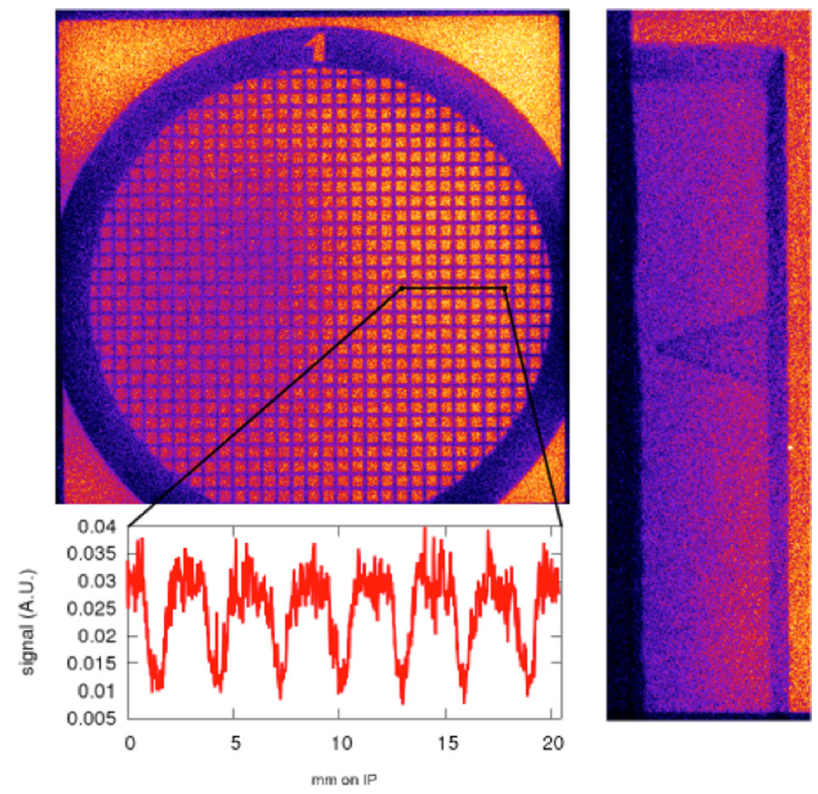

FIG. 4. X-ray point projection radiography calibration images: (left) Au grid $20 \mu \mathrm{m}$ thick with $60 \mu \mathrm{m}$ spacing; (right) buried cone target. The black area in (c) corresponds to a tungsten shield, which was set up to avoid direct Xray irradiation produced by the $\mathrm{ns}$ beam. The shadow of the $\mathrm{Cu}$ layer is larger than $10 \mu \mathrm{m}$ due to the relative angle with the wire backlighter, aligned to the cone tip.

Bremsstrahlung emission. The red line in Fig. 3 corresponds to such spectrum, while the dashed blue line shows what would be obtained with $\mathrm{K} \alpha$ emission only (i.e., using the mass absorption coefficient for $\mathrm{Al}$ at $8 \mathrm{keV}$ of $50.33 \mathrm{~cm}^{2} / \mathrm{g}$ as provided by $\mathrm{NIST}^{21}$ ). The two differ only by a constant, representing the contribution of the Bremsstrahlung x-ray emission, whose value is $0.0056 \pm 0.0006$ PSL. We performed also some numerical simulations with the code with FLYCHK $^{22}$ to obtain a synthetic x-ray spectrum composed of two peaks ( $\mathrm{K} \alpha 1$ and $\mathrm{K} \alpha 2$ emission) and a Bremsstrahlung term. In all cases, data for mass attenuation coefficients were obtained from Refs. 16 and 23 while the sensitivity of the IP was taken from Ref. 24.

For the experiment, we used a $\mathrm{Cu}$ wire target because $8 \mathrm{keV}$ photons can penetrate through the thick $\mathrm{C}$ target (about $1 \mathrm{~mm}$ thick) while still provide sufficient contrast to detect a shocked region which, a-priori, is $\sim 500 \mu \mathrm{m}$ wide and corresponds to a density jump of $\sim 2 \times$.

We also experimentally measured the spatial resolution of the x-ray radiography imaging system. Fig. 4 shows static radiography images of a Au grid and a standard buried cone target identical to those used in the experiment. The Au grid is $20 \mu \mathrm{m}$ thick with $60 \mu \mathrm{m}$ spacing, the spatial resolution obtained from the grid calibration radiography is $\sim 11 \mu \mathrm{m}$ using a $10 \mu \mathrm{m} \mathrm{Cu}$ wire. This value is estimated as the $10 \%$ to 90\% distance in the intensity lineout in Figure 4(b).

\section{APPLICATION OF X-RAY POINT PROJECTION RADIOGRAPHY TO FAST IGNITION}

As already mentioned above, in fast electron FI, the survival of the cone structure undergoing the extreme pressures generated during the capsule implosion is of vital importance for the success of this scheme. X-ray point projection 


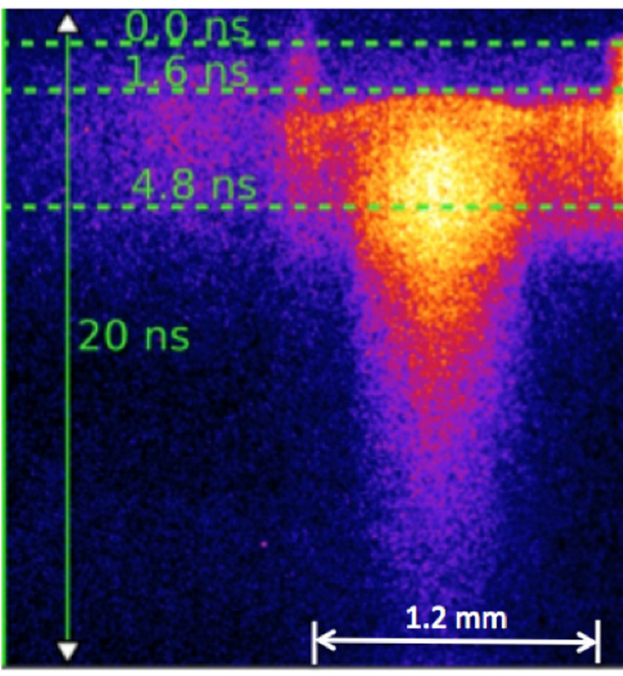

FIG. 5. Shot on a $40 \mu \mathrm{m} \mathrm{Al} \mathrm{target} \mathrm{(energy:} 574 \mathrm{~J}$ ). Breakout time: $1.6 \mathrm{~ns}$.

radiography can provide information on the cone structure condition at different time delays. The observations are supported by SOP data providing the shock breakout time from the cone tip.

The SOP measurements were performed using an Hamamatsu C 7700 streak camera, synchronized with the arrival time of the ns pulse. Figs. 5 and 6 represent respectively the SOP calibration data obtained on a $40 \mu \mathrm{m} \mathrm{Al} \mathrm{target} \mathrm{and} \mathrm{a}$ SOP acquisition from a buried cone target. The temporal window is respectively 20 and $50 \mathrm{~ns}$ for the two acquisitions, resulting in a temporal resolution, using a $300 \mu \mathrm{m}$ slit, of respectively 250 and 600 ps.

The shock breakout times, measured from the arrival of laser pulse on target, are $1.6 \mathrm{~ns}$ for the $\mathrm{Al} 40 \mu \mathrm{m}$ target (574 J in $4.7 \mathrm{~ns}$ ) and $8.2 \mathrm{~ns}$ for the cone target $(521 \mathrm{~J}$ in $4.2 \mathrm{~ns})$. The average shock velocity resulting from shock breakout times from buried cone targets was $\mathrm{D}=17 \pm 1.8 \mu \mathrm{m} / \mathrm{ns}$. The flat target was used as reference to validate the ns pulse energy used in the hydrodynamic simulations.

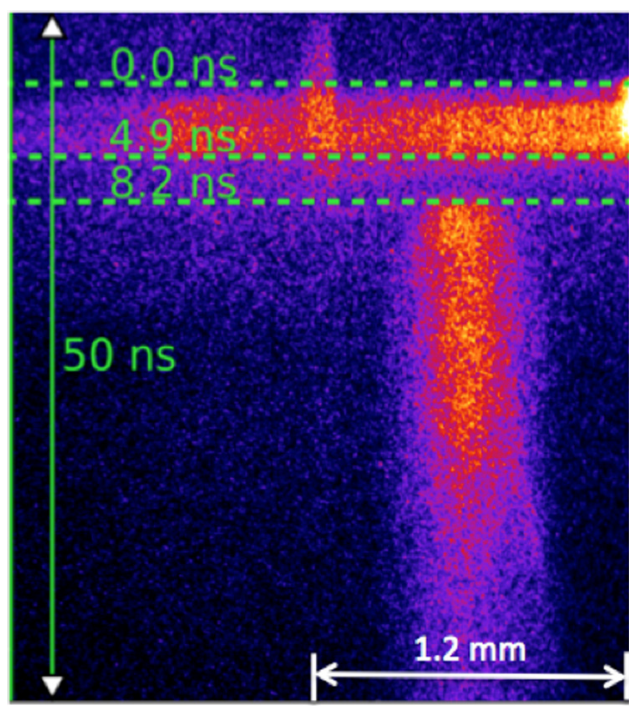

FIG. 6. Shot on a cone target (energy: $521 \mathrm{~J}$ ). Breakout time: $8.2 \mathrm{~ns}$.
Of course this is just a value averaged over the different layers of the target (polystyrene $6.5 \mu \mathrm{m}$, Silver $9.8 \mu \mathrm{m}$, porous carbon $100 \mu \mathrm{m}$, and copper $23 \mu \mathrm{m}$ ).

The $\mathrm{x}$-ray radiography images of shock propagation show that the cone structure seems to survive the shock solicitation. Fig. 7 shows a series of x-ray radiography images of shock propagation at different time delays. Unfortunately in many of these acquisitions, the $\mathrm{Cu}$ wire had to be replaced by a $10 \mu \mathrm{m}$ thick $\mathrm{Cu}$ foil, disposed normally to the cone target axis. This choice was made due to the shot-to-shot pointing fluctuation of the LULI2000 ps pulse, larger than $10 \mu \mathrm{m}$, which made the backlighter performances very unstable. Note that, the $10 \mu \mathrm{m} \mathrm{Cu}$ foil guarantees the same resolution along the cone axis than the $\mathrm{Cu}$ wire while the resolution along the axis normal is reduced $(\sim 50 \mu \mathrm{m})$. Indeed the source size instead of being related to the wire diameter is now related to the $\mathrm{Cu}-\mathrm{K} \alpha$ spot size, which always appears much larger than the laser spot. $^{25}$ Nevertheless, this still allows to clearly identify the cone features as shown in Fig. 7. Here, for delays larger than $8.2 \mathrm{~ns}$, consistently with shock breakout measurements from SOP, we see that the shock is propagating in the cone region but that the overall structure of the cone is still visible implying cone survival. This result is in line with the cone-shell implosion radiography results recently described in Ref. 17 , showing substantial survival of the cone at implosion bang time.

\section{APPLICATION OF X-RAY POINT PROJECTION RADIOGRAPHY TO STUDY EOS (OF CARBON)}

The $\mathrm{x}$-ray point projection radiography allows not only to measure the position of the shock but also, simultaneously that of the $\mathrm{Ag} / \mathrm{C}$ interface. The data points for shock front position and $\mathrm{Ag}$ layer position as function of time are displayed in Fig. 8 together with the results from hydrodynamic simulations performed with the $1 \mathrm{D}$ code $\mathrm{MULTI}^{26}$ using opacity tables calculated using the atomic physics code SNOP. ${ }^{27}$ The SOP measurement on the $40 \mu \mathrm{m}$ Al layer was used as a reference to calibrate the laser intensity corresponding to a specific laser energy used in the hydrodynamic simulations, In this figure, the points represent the experimental data while the colored areas represent the MULTI simulations. The areas reflect the shot-to-shot laser energy fluctuation. In general, the $\mathrm{Ag} / \mathrm{C}$ interface is clearly visible in all images (due to the high opacity of the Ag layer) while the position of the shock front, corresponding to a smaller density jump, is identified with more difficulties. The shock breakout timing obtained from the hydrodynamic simulations is in very good agreement with the experimental data.

We see that, despite experimental uncertainties, the hydrodynamic simulations can well reproduce the experimental values for shock front and Ag layer position, which is also a good validation for code behavior in such complex situation. We also see that, after an initial phase, characterized by almost constant velocities, the shock front decelerates and also the fluid velocity decreases with time.

If we consider only the initial phase $(t<15 \mathrm{~ns}$, since the $\mathrm{Ag} / \mathrm{C}$ interface velocity slows down for longer delays), we can interpolate the experimental points giving the shock 


\section{SHOT $\mathrm{N}^{\circ} 41$}

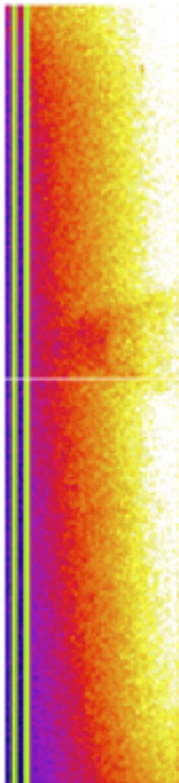

$\Delta \mathrm{t}=0 \mathrm{~ns}$

$E_{\text {nano }}=0 \mathrm{~J}$
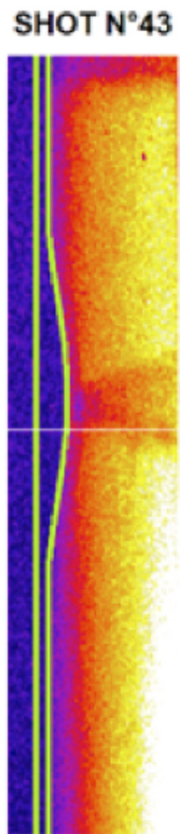

\section{$\Delta \mathrm{t}=9 \mathrm{~ns}$}
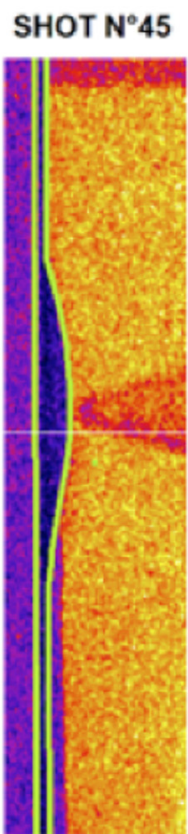

$\Delta \mathrm{t}=11 \mathrm{~ns}$
SHOT N ${ }^{\circ} 46$

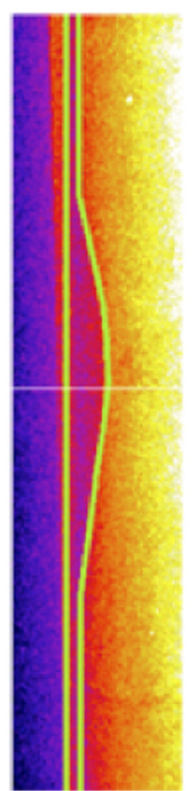

$\Delta \mathrm{t}=13 \mathrm{~ns}$
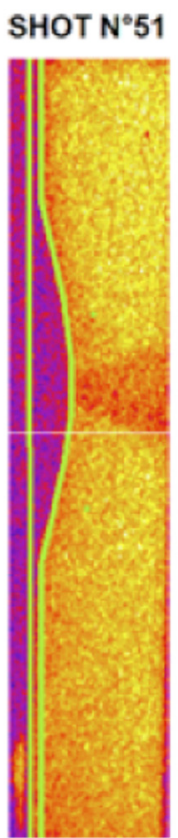

$\Delta \mathrm{t}=15 \mathrm{~ns}$
SHOT $N^{\circ} 52$

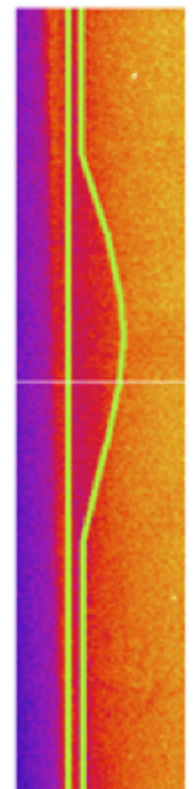

$\Delta \mathrm{t}=17 \mathrm{~ns}$
FIG. 7. X-ray radiography of the coneshaped target after interaction with the long pulse laser coming from the left in these pictures. The laser is coming from the left. The two lines represent the target surface and the position of the $\mathrm{Ag} / \mathrm{C}$ interface at various times. front and the interface position with straight lines. These then provides the shock velocity $\mathrm{D}$ and the fluid velocity $\mathrm{U}$ (equal to the velocity of interface) in carbon aftershock passage. For the data in Fig. 8, we get

$$
\mathrm{U}=8.78 \pm 0.68 \mu \mathrm{m} / \mathrm{ns} \quad \mathrm{D}=20.80 \pm 2.50 \mu \mathrm{m} / \mathrm{ns} .
$$

Given the Hugoniot-Rankine equation set, the pressure and density of the shocked material can be calculated as

$$
\rho=\rho_{\mathrm{o}} \mathrm{D} /(\mathrm{D}-\mathrm{U}) \quad \mathrm{P}=\rho_{\mathrm{o}} \mathrm{DU},
$$

where $\rho_{0}$ is the unperturbed density and the unperturbed pressure was set equal to zero.

The calculated shocked density and pressure are

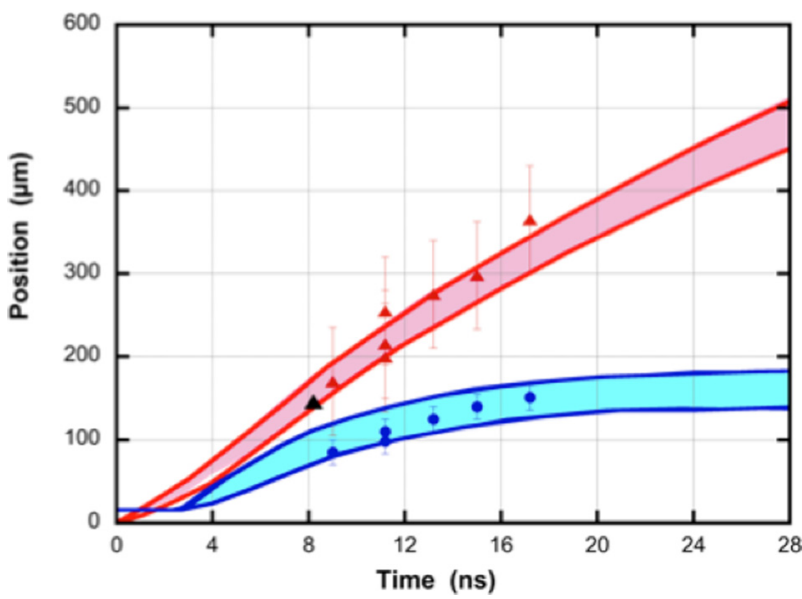

FIG. 8. Experimental results and MULTI simulations for the shock propagation (in red) and the silver layer displacement (in blue), SOP breakout time is displayed in black. The stripes represent the lower and upper simulated value, accounting for the $20 \%$ shot-to-shot energy fluctuation in the experiment.

$$
\rho=3.46 \pm 0.36 \mathrm{~g} / \mathrm{cm}^{3} \quad \mathrm{P}=3.65 \pm 0.52 \mathrm{MBar} .
$$

This method then allows obtaining a point on the Shock Polar of Porous Carbon. This point is shown in Fig. 9, together with three curves, relative to three different equations of state. The blue curve is obtained from the $\mathrm{SESAME}^{28}$ table for Carbon 7833, preheated at $0.5 \mathrm{eV}$ to account for the initial density of $2 \mathrm{~g} / \mathrm{cm}^{3}$ of porous carbon. The red curve is calculated with MPQEOS ${ }^{29}$ for porous carbon, with an initial density of $2 \mathrm{~g} / \mathrm{cc}$ and an initial temperature of $0.025 \mathrm{eV}$. In order to account for the reduced density of carbon, we have changed its bulk modulus following what described in Ref. 30. Finally, for comparison, we have also drawn the green curve (MPQEOS with initial conditions $2 \mathrm{~g} / \mathrm{cc}$ and $0.5 \mathrm{eV}$ ) to show the effect of preheating on the Hugoniot curve.

Fig. 10 shows the same curves and the same experimental point in the $\rho$-P plane. In this case, the agreement

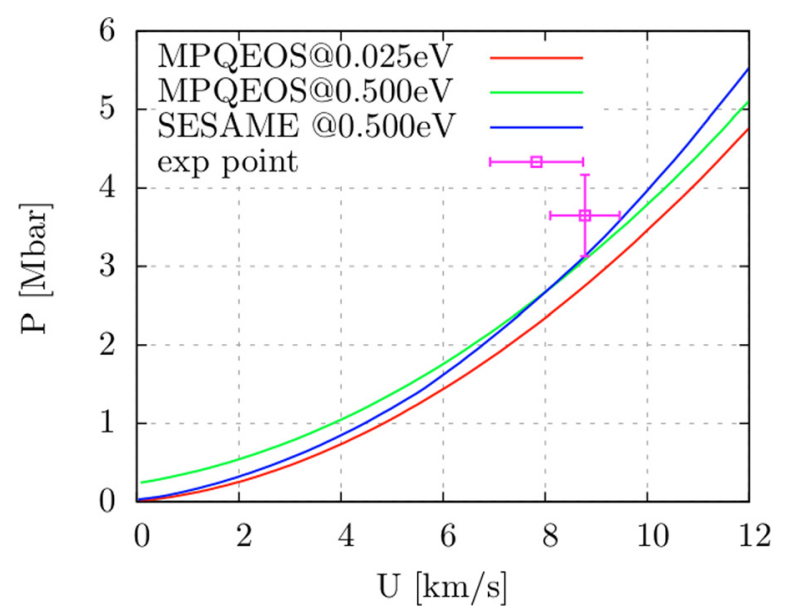

FIG. 9. Comparison between the shock polar curves from different EoS and the experimental point for Porous Carbon. 


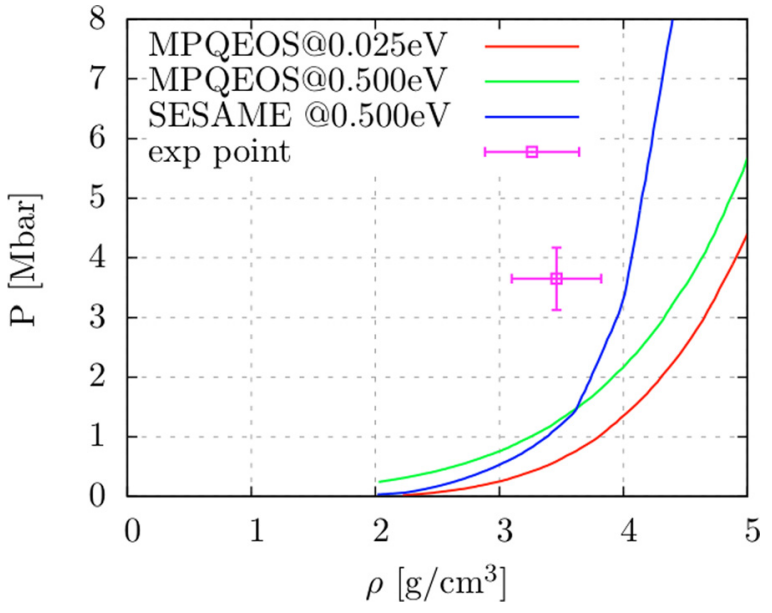

FIG. 10. Comparison between the Hugoniot curves traced with different EoS and the experimental point for Porous Carbon.

between the calculated value and the EOS curves is somewhat poorer (this is indeed expected and a common feature of shock EOS experiment due to the non-linearity of Hugoniot-Rankine relations).

The overall best agreement, considering the U-P and the $\rho$-P planes, is found with the SESAME curve with $0.5 \mathrm{eV}$ initial temperature. However, we must be careful in the conclusion because in reality, the velocities measured in Figure 8 are mean velocities, which are affected by the shotto-shot laser energy fluctuation.

Since these measurements, as we mentioned before, represented the measurement of shock propagation in the target in preparation of a following experiment, the ns laser energy was kept constant, allowing for the determination of one point only on the shock polar of Porous Carbon. However, this method can in principle be used to complement those already described in literature, which have provided EOS data for an element like carbon, which is very important for astrophysical and planetological applications. ${ }^{25,31-33}$

Let us notice that $\mathrm{x}$-ray radiography techniques have already been applied in the past to study the EOS of materials in the Megabar pressure range, first for the study of EOS of deuterium $^{34}$ and then for low-Z materials as plastics and beryllium. ${ }^{35}$ In this cases, a ns x-ray backlighter was associated to a streak camera allowing following continuously the evolution of shock and fluid velocity in time, in a single laser shot. Of course such technique is less time-consuming (D and $U$ being determined in a single shot) and less prone to laser energy fluctuations. On the other side, they do not allow checking the planarity of the shock front in each laser shot and are more sensitive to misalignment problems (in our technique indeed it is always possible to select the central, planar, part of the shock front and measure its position). Also, we notice that progress in laser reliability in ns laser systems (see for instance the performances of the National ignition Facility) really allow to exactly get the same laser pulse characteristics on different laser shots, while, on the other side, fluctuations in the ps laser beam are not so important since they will mainly affect the x-ray backlighter level. On the other side, one should also notice that recently x-ray
Thomson scattering has provided useful information on EOS of materials. ${ }^{36}$ This is certainly an important technique, which however, in our opinion, cannot be separated by an independent and simultaneous determination of more classical parameters like shock and fluid velocity.

Let us finally notice that the result shown in Figs. 9 and 10 would imply a compressibility of carbon, which is smaller than what implied by the SESAME tables. This is in contrast, for instance, with the results presented in Ref. 33. However, again, we repeat that the goal of the present work was more a "proof of principle" than a systematic study of EOS.

\section{CONCLUSION}

In this work, we presented an advanced x-ray radiography technique for HED research. The technique allows to obtain 2D spatially resolved images of the plasma density profiles at different time delays, providing information on several aspects of the shock dynamics. A remarkable agreement was obtained between experimental results and predictions of hydro codes.

In particular, the radiographic technique allowed, at the same time, to obtain information about the cone target survival under the action of a strong shock and to measure a point in the equation of state of porous Carbon. Although other radiographic techniques can provide similar information, as described in the introduction, the one presented here allows for applications in several small and medium size laser facilities where smaller laser energies are available. The possible applications range from time resolved imaging of ultrafast physical phenomena to the determination of EoS of materials at high pressures. The relatively simple setup allows implementing this technique on a wide variety of experimental designs, therefore representing a valuable diagnostic for HEDP studies.

\section{ACKNOWLEDGMENTS}

The work presented here was performed under financial support from both the French National Agency for Research (ANR) and the competitiveness cluster Alpha - Route des Lasers through project TERRE ANR-2011-BS04-014. This study has been carried out in the frame of "the Investments for the future" Programme IdEx Bordeaux-LAPHIA (ANR-10-IDEX-03-02). The authors acknowledge LULI for giving access to the LULI2000 facility in the context of the preparatory phase of the HiPER project (Work Package 10: experimental validation program), as well as the support of the LULI2000 engineering staff in designing the setup implementation and during the experimental run. This work was also partially supported by the Italian MIUR project PRIN 2009FCC9MS. This work has been carried out within the framework of the EUROfusion Consortium and has received funding from the European Union's Horizon 2020 research and innovation programme under grant agreement number 633053. The views and opinions expressed herein do not necessarily reflect those of the European Commission.

${ }^{1}$ R. P. Drake, High-Energy-Density Physics: Fundamentals, Inertial Fusion, and Experimental Astrophysics (Springer, 2006). 
${ }^{2}$ B. A. Remington, R. P. Drake, and D. D. Ryutov, Rev. Modern Phys. 78(3), 755 (2006).

${ }^{3}$ S. H. Glenzer and R. Redmer, Review of Modern Physics 81(4), 1625 (2009). ${ }^{4}$ J. Lindl, Phys. Plasmas 2, 3933-4024 (1995).

${ }^{5}$ W. B. Hubbard, W. J. Nellis, A. C. Mitchell, N. C. Holmes, S. S. Limaye, and P. C. McCandless, Science 253, 648-651(1991).

${ }^{6}$ J. D. Lindl, R. L. McCrory, and E. M. Campbell, Phys. Today 45(9), 32 (1992).

${ }^{7}$ M. Tabak, J. Hammer, M. E. Glinsky, W. L. Kruer, S. C. Wilks, J. Woodworth, E. M. Campbell, M. D. Perry, and R. J. Mason, Phys. Plasmas 1, 1626 (1994).

${ }^{8}$ R. Betti, C. D. Zhou, K. S. Anderson, L. J. Perkins, W. Theobald, and A. A. Solodov, Phys. Rev. Lett. 98(15), 155001 (2007).

${ }^{9}$ R. Kodama, P. A. Norreys, K. Mima, A. E. Dangor, R. G. Evans, H. Fujita, Y. Kitagawa, K. Krushelnick, T. Miyakoshi, N. Miyanaga, T. Norimatsu, S. J. Rose, T. Shozaki, K. Shigemori, A. Sunahara, M. Tampo, K. A. Tanaka, Y. Toyama, Y. Yamanaka, and M. Zepf, Nature 412, 798 (2001).

${ }^{10}$ S. Fujioka, Z. Zhang, N. Yamamoto, S. Ohira, Y. Fujii, K. Ishihara, T. Johzaki, A. Sunahara, Y. Arikawa, K. Shigemori, Y. Hironaka, Y. Sakawa, Y. Nakata, J. Kawanaka, H. Nagatomo, H. Shiraga, N. Miyanaga, T. Norimatsu, H. Nishimura, and H. Azechi, Plasma Phys. Controlled Fusion 54, 124042 (2012).

${ }^{11}$ M. H. Key, J. C. Adam, K. U. Akli, M. Borghesi, M. H. Chen, R. G. Evans, R. R. Freeman, H. Habara, S. P. Hatchett, J. M. Hill, A. Heron, J. A. King, R. Kodama, K. L. Lancaster, A. J. MacKinnon, P. Patel, T. Phillips, L. Romagnani, R. A. Snavely, R. Stephens, C. Stoeckl, R. Town, Y. Toyama, B. Zhang, M. Zepf, and P. A. Norreys, Phys. Plasmas 15, 022701 (2008).

${ }^{12}$ A. Debayle, J. J. Honrubia, E. D'Humières, V. T. Tikhonchuk, S. Micheau, and M. Geissler, J. Phys.: Conf. Ser. 244(2), 022032 (2010).

${ }^{13}$ L. H. Barker and R. Hollenbach, J. Appl. Phys. 43, 4669 (1972).

${ }^{14}$ S. Laffite, S. D. Baton, P. Combis, J. Clerouin, M. Koenig, V. Recoules, C. Rousseaux, and L. Videau, "Velocity Interferometer blanking due to preheating in a double pulse planar experiment," Physics of Plasmas 21, 082705 (2014).

${ }^{15}$ J. D. Kilkenny, S. G. Glendinning, S. W. Haan, B. A. Hammel, J. D. Lindl, D. Munro, B. A. Remington, S. V. Weber, J. P. Knauer, and C. P. Verdon, Phys. Plasmas 1, 1379 (1994).

${ }^{16}$ F. J. Marshall and G. R. Bennett, Rev. Sci. Instrum. 70, 617 (1999).

${ }^{17}$ F. J. Marshall, P. W. McKenty, J. A. Delettrez, R. Epstein, J. P. Knauer, V. A. Smalyuk, J. A. Frenje, C. K. Li, R. D. Petrasso, F. H. Séguin, and R. C. Mancini, Phys. Rev. Lett. 102, 185004 (2009).

${ }^{18}$ W. Theobald, A. A. Solodov, C. Stoeckl, V. Yu. Glebov, S. Ivancic, F. J. Marshall, G. McKiernan, C. Mileham, T. C. Sangster, F. N. Beg, C. Jarrott, E. Giraldez, R. B. Stephens, M. S. Wei, M. H. Key, H. McLean, and J. Santos, in 54th Annual Meeting of the APS Division of Plasma Physics, Providence, Rhode Island, 29 October-2 November 2012, Vol. 57, p. 12; available at http://meetings.aps.org/link/BAPS.2012.DPP.GO5.4.
${ }^{19}$ R. Tommasini, S. P. Hatchett, D. S. Hey, C. Iglesias, N. Izumi, J. A. Koch, O. L. Landen, A. J. MacKinnon, C. Sorce, J. A. Delettrez, V. Yu. Glebov, T. C. Sangster, and C. Stoeckl, Phys. Plasmas 18, 056309 (2011).

${ }^{20}$ V. M. Ovchinnikov, D. W. Schumacher, M. McMahon, E. A. Chowdhury, C. D. Chen, A. Morace, and R. R. Freeman, Phys. Rev. Lett. 110(6), 065007 (2013).

${ }^{21}$. J. H. Hubbell and S. M. Seltzer, National Institute of Standards and Technology (1989).

${ }^{22}$ H.-K. Chung, M. H. Chen, W. L. Morgan, Y. Ralchenko, and R. W. Lee, High Energy Density Phys. 1, 3-12 (2005).

${ }^{23}$ B. L. Henke, E. M. Gullikson, and J. C. Davis. At. Data Nucl. Data Tables 54(2), 181-342 (1993).

${ }^{24}$ T. Doppner, E. L. Dewald, L. Divol, C. A. Thomas, S. Burns, P. M. Celliers, N. Izumi, J. L. Kline, G. LaCaille, J. M. McNaney, R. R. Prasad, H. F. Robey, S. H. Glenzer, and O. L. Landen, Rev. Sci. Instrum. 83(10), 10508 (2012).

${ }^{25}$ R. B. Stephens et al., Phys. Rev. E 69, 066414 (2004).

${ }^{26}$ R. Ramis, R. Schmalz, and J. Meyer-Ter-Vehn, Comput. Phys. Commun. 49, 475 (1988)

${ }^{27} \mathrm{~K}$. Eidmann, "Radiation transport and atomic physics modeling in high energy density laser-produced plasmas," Laser Part. Beams 12, 223-244 (1994).

${ }^{28}$ Los Alamos National Laboratory EOS Database, edited by S. P. Lyon and J. D. Johnson (1992) Report LA-UR-92-3407, Table Hydr5251.

${ }^{29}$ A. J. Kemp and J. Meyer-ter-Vehn, Nucl. Instrum. Methods Phys. Res. Sec. A 415(3), 674-676 (1998).

${ }^{30}$ S. Paleari, D. Batani, T. Vinci, R. Benocci, K. Shigemori, Y. Hironaka, T. Kadono, A. Shiroshita, P. Piseri, S. Bellucci, A. Mangione, and A. Aliverdiev, Eur. J. Phys. 67, 136 (2013).

${ }^{31}$ K. Falk, E. J. Gamboa, G. Kagan, D. S. Montgomery, B. Srinivasan, and J. F. Benage, Phys. Rev. Lett. 112, 155003 (2014).

${ }^{32}$ D. Batani, S. Paleari, T. Vinci, R. Benocci, K. Shigemori, Y. Hironaka, T. Kadono, and A. Shiroshita, Laser Part. Beams 31, 457-464 (2013).

${ }^{33}$ D. Batani, H. Stabile, M. Tomasini, G. Lucchini, A. Ravasio, M. Koenig, A. Benuzzi-Mounaix, H. Nishimura, Y. Ochi, J. Ullschmied, J. Skala, B. Kralikova, M. Pfeifer, Ch. Kadlec, T. Mocek, A. Präg, T. Hall, P. Milani, E. Barborini, and P. Piseri, Phys. Rev. Lett. 92, 065503 (2004).

${ }^{34}$ L. B. Da Silva, P. Celliers, G. W. Collins, K. S. Budil, N. C. Holmes, T. W. Barbee, Jr., B. A. Hammel, J. D. Kilkenny, R. J. Wallace, M. Ross, R. Cauble, A. Ng, and G. Chiu, Phys. Rev. Lett. 78, 483 (1997).

${ }^{35}$ R. Cauble, T. S. Perry, D. R. Bach, K. S. Budil, B. A. Hammel, G. W. Collins, D. M. Gold, J. Dunn, P. Celliers, L. B. Da Silva, M. E. Foord, R. J. Wallace, R. E. Stewart, and N. C. Woolsey, Phys. Rev. Lett. 80, 1248 (1998).

${ }^{36}$ A. L. Kritcher, P. Neumayer, J. Castor, T. Döppner, R. W. Falcone, O. L. Landen, H. Ja Lee, R. W. Lee, E. C. Morse, A. Ng, S. Pollaine, D. Price, and S. H. Glenzer, Science 322, 69 (2008). 